\title{
PROBLEMA DE VALOR DE CONTORNO DA GEODÉSIA: UMA ABORDAGEM CONCEITUAL
}

\author{
Geodetic Boundary Value Problem: A conceptual approach \\ GABRIEL DO NASCIMENTO GUIMARÃES ${ }^{1}$ \\ DENIZAR BLITZKOW ${ }^{1}$ \\ ${ }^{1}$ Departamento de Engenharia de Transportes da \\ Escola Politécnica da Universidade de São Paulo - EPUSP \\ LTG - Laboratório de Topografia e Geodésia \\ gabrielguimaraes33@hotmail.com; dblitzko@usp.br
}

\begin{abstract}
RESUMO
Um dos problemas da Geodésia é determinar o campo de gravidade externo às massas, além da superfície limitante, bem como a variação temporal do mesmo. Stokes propôs no século XVIII uma formulação para a solução desse problema, porém implicava em algumas dificuldades. A formulação proposta por Molodensky em meados do século XX abriu uma nova perspectiva para a solução do problema. Ao longo dos anos, na Geodésia, a relevância do Problema de Valor de Contorno da Geodésia (PVCG) tem sido reconhecida como base teórica para essa disciplina. Além disso, o PVCG ganhou novo impulso com a era espacial e com o passar dos anos algumas alternativas foram elaboradas (super determinado, misto, solução de Brovar). Este trabalho tem como objetivo retratar as formulações de Stokes e Molodensky, bem como as principais alternativas do PVCG.

Palavras-chave: Geodésia; Problema de Valor de Contorno; Superfície limitante.
\end{abstract}

\section{ABSTRACT}

One of the Geodesy's problems is to determine the masses external gravity field, beyond the boundary surface, as well as the temporal variation. Stokes proposed a formulation in the $18^{\text {th }}$ century to solve this problem, however some problems were implied. The formulation proposed by Molodensky in the middle of the $-20^{\text {th }}$ century opened a new perspective to solve this problem. Throughout the years, in Geodesy, the relevance of the Boundary Value Problem of Geodesy (BVPG) has been recognized as a theoretical basis for that discipline. In addition, the BVPG gained new impulse with the spatial era and in the maentime, many alternatives 
have been developed (overdetermined, mixed, Brovar's solution). This paper aims at presenting Stokes' and Molodensky's formulations as well as the main BVPG alternatives.

Keywords: Geodesy; Boundary Value Problem; Boundary surface.

\section{INTRODUÇÃO}

A componente altimétrica sempre foi uma das preocupações da Geodésia. Com efeito, o advento da era espacial tornou possível obter as coordenadas cartesianas $(X, Y, Z)$ que podem ser transformadas em coordenadas geodésicas $(\varphi, \lambda$, h). Anteriormente, as componentes planimétricas eram estabelecidas a partir da triangulação e poligonação e a componente altimétrica por meio de nivelamento geométrico, o que ocasionava uma dicotomia entre as redes planimétrica e altimétrica. Hoje, a altitude elipsoidal também pode ser determinada a partir de observações de satélites e está referenciada a um modelo geométrico utilizado para os cálculos geodésicos (elipsoide) e que não possui um significado físico. Isso faz com que essa componente não atenda a uma pequena quantidade de tarefas da Engenharia. Logo, percebe-se a necessidade de uma altitude adequada às exigências usuais.

O estabelecimento de um referencial altimétrico está associado à definição e à realização do mesmo. A escolha de uma superfície de referência adequada juntamente com um sistema de altitude constitui a definição de um referencial altimétrico. A realização pode ser conduzida através da determinação do nível médio dos mares durante um determinado período a partir de observações maregráficas. Além disso, deve-se efetuar correções referentes à carga oceânica e atmosférica, ao movimento da crosta terrestre e às marés oceânicas. Na Geodésia, as altitudes se classificam segundo a sua determinação, a aplicação e o modelo matemático ou físico considerado na sua definição. Desse modo, elas se distinguem em altitudes com característica geométrica e altitudes com característica física. Além do auxílio das técnicas espaciais, a altitude de um ponto também é obtida a partir de um procedimento conhecido como nivelamento. Esse pode ser barométrico, trigonométrico ou geométrico.

Listing, em 1873, caracterizou a forma da Terra como sendo um geoide. A superfície geoidal é equipotencial do campo de gravidade coincidente com o nível médio dos mares não perturbado em um dado instante. Uma das preocupações da Geodésia é o estudo do geoide e sua determinação realizada por meio do conhecimento do campo de gravidade que envolve a distribuição de massa e o efeito rotacional da Terra e que permite deduzir a sua forma. Para se determinar a função potencial do referido campo é necessário envolver o que se denomina de "Problema de Valor de Contorno da Geodésia (PVCG)" (FREITAS; BLITZKOW, 1999).

Existe uma relação direta entre o conceito de altitude e o PVCG, a qual deve ser analisada tanto do ponto de vista geométrico quanto físico. Contudo, deve-se 
levar em conta a superfície eleita como origem para as medições altimétricas, além do seu significado como superfície de referência do campo de gravidade. Ademais, é necessário considerar os efeitos seculares e periódicos sobre as observações maregráficas na materialização da superfície de referência (BLITZKOW; CAMPOS; FREITAS, 2007).

\section{A TEORIA DO POTENCIAL}

A atração física que um determinado corpo exerce sobre objetos próximos a ele é o resultado da força gravitacional. Isaac Newton formulou a Lei Universal da Gravitação em sua obra Philosophiae Naturalis Principia Mathematica, publicada em 1687, que descreve também as Leis de Newton - leis do movimento dos corpos celestes, base da mecânica clássica.

Duas partículas se atraem mutuamente com uma força proporcional ao produto de suas massas e inversamente proporcional ao quadrado da distância que as separa:

$$
F=G \frac{m_{1} m_{2}}{l^{2}}
$$

onde $\mathrm{G}$ é a constante universal da gravitação, cujo valor no Sistema Internacional (SI) é:

$$
G=(6,67384 \pm 0,00080) * 10^{-11} \mathrm{~m}^{3} \mathrm{~kg}^{-1} \mathrm{~s}^{-2}
$$

É conveniente, para evitar possível ambiguidade, considerar uma das partículas como "atrativa" e outra como "atraída", atribuindo massa unitária à última. Desta forma:

$$
F=G \frac{m}{l^{2}}
$$

e expressa a atração gravitacional, gerada pela partícula de massa $m$, que atua sobre uma massa unitária situada a uma distância $l$. O potencial gravitacional de atração ou newtoniano é uma função escalar definida por:

$$
V=G \frac{m}{l}
$$

No caso de um sistema discreto de partículas:

$$
V=G \sum_{i=1}^{n} \frac{m_{i}}{l_{i}}
$$


Introduzindo um sistema de coordenadas cartesianas, considerando o potencial de atração $\mathrm{V}$, as correspondentes derivadas parciais de primeira ordem são (GEMAEL, 1999):

$$
\begin{aligned}
& \frac{\partial V}{\partial x}=-G \sum_{i=1}^{n} \frac{m_{i}\left(x-x_{i}^{\prime}\right)}{l_{i}^{3}}, \\
& \frac{\partial V}{\partial y}=-G \sum_{i=1}^{n} \frac{m_{i}\left(y-y_{i}^{\prime}\right)}{l_{i}^{3}}, \\
& \frac{\partial V}{\partial z}=-G \sum_{i=1}^{n} \frac{m_{i}\left(z-z_{i}^{\prime}\right)}{l_{i}^{3}}
\end{aligned}
$$

e as de segunda ordem:

$$
\begin{aligned}
\frac{\partial^{2} V}{\partial x^{2}} & =G \sum_{i=1}^{n} \frac{m_{i}}{l_{i}^{3}}-3 G \sum_{i=1}^{n} \frac{m_{i}\left(x-x_{i}^{\prime}\right)^{2}}{l_{i}^{5}} \\
\frac{\partial^{2} V}{\partial y^{2}} & =G \sum_{i=1}^{n} \frac{m_{i}}{l_{i}^{3}}-3 G \sum_{i=1}^{n} \frac{m_{i}\left(y-y_{i}^{\prime}\right)^{2}}{l_{i}^{5}} \\
\frac{\partial^{2} V}{\partial z^{2}} & =G \sum_{i=1}^{n} \frac{m_{i}}{l_{i}^{3}}-3 G \sum_{i=1}^{n} \frac{m_{i}\left(z-z_{i}^{\prime}\right)^{2}}{l_{i}^{5}}
\end{aligned}
$$

somando membro a membro a equação (6) tem-se:

$$
\frac{\partial^{2} V}{\partial x^{2}}+\frac{\partial^{2} V}{\partial y^{2}}+\frac{\partial^{2} V}{\partial z^{2}}=3 \sum_{i=1}^{n} \frac{m_{i}}{l_{i}^{3}}-3 \sum_{i=1}^{n} \frac{m_{i}}{l_{i}^{3}}=0
$$

ou, simplesmente:

$$
\nabla^{2} V=0
$$

A equação (8) é conhecida como equação de Laplace. Ela traduz o fato de que o laplaciano do potencial gravitacional é nulo fora das massas atrativas. Por essa razão ela é denominada função harmônica, pois satisfaz a equação de Laplace em todos os pontos no exterior do corpo.

\section{O PROBLEMA DE VALOR DE CONTORNO DA GEODÉSIA}

O conhecimento de valores de uma determinada variável sobre uma superfície limitante e a partir daí, a determinação de uma função que se relacione àquela 
variável, conduz ao que é chamado de "problema de valor de contorno" (BLITZKOW, 1996). Em suma, no PVC o que se busca determinar é uma função para um dado corpo.

O problema de valor de contorno é determinar o campo de gravidade externo às massas sendo que a superfície limitante é desconhecida. Stokes propôs uma formulação para obter o potencial perturbador em função da anomalia de gravidade sobre a superfície geoidal. Entretanto, essa proposição implica em algumas dificuldades, pois é um problema interno às massas. Uma nova formulação do problema fundamental da Geodésia foi proposta por Molodensky. Trata-se de um problema externo às massas que utiliza a superfície física como limite. Com isso não é necessário o conhecimento, mesmo que aproximado, de um modelo de distribuição de densidade no interior da crosta entre a superfície física e o geoide. No entanto, essa superfície não tem o mesmo significado físico que a superfície geoidal, pois não é uma superfície equipotencial.

A teoria do potencial apresenta três problemas de valor de contorno:

- Primeiro problema (Dirichlet): a partir do conhecimento dos valores de uma função $T$ sobre uma superfície $S$, determina-se essa função $T$ de tal maneira que ela seja harmônica no interior ou no exterior de $S$ quando são conhecidos os valores que a função assume sobre a superfície:

$$
\begin{cases}\Delta T=0 & r>R \\ T=\bar{T} & r=R\end{cases}
$$

- Segundo problema (Neumann): a partir do conhecimento dos valores da derivada normal da função T sobre a superfície $S, \partial T / \partial r$, determina-se a função $T$ de modo que ela seja harmônica interna ou externamente a $S$ :

$$
\begin{cases}\Delta T=0 & r>R \\ \frac{\partial T}{\partial r}=-\delta_{g} & r=R\end{cases}
$$

- Terceiro problema (Hilbert): a partir dos valores da combinação linear da função $T$ com sua derivada normal sobre a superfície $S$, determina-se a função $T$ nas condições anteriores:

$$
\begin{cases}\Delta T=0 & r>R \\ \frac{\partial T}{\partial r}+\frac{2}{r} T=-\Delta g & r=R\end{cases}
$$


Tais problemas não apresentam solução única, pois infinitas distribuições de massa podem gerar o mesmo potencial. O terceiro problema tem relevância para a Geodésia, uma vez que a determinação do potencial perturbador de certa forma se relaciona a ele. A anomalia de gravidade é representada como uma combinação linear entre o potencial perturbador e sua derivada normal e é expressa por uma equação diferencial conhecida como "equação fundamental da Geodésia":

$$
\Delta g=-\frac{\partial T}{\partial h}+\frac{1}{\gamma} \frac{\partial \gamma}{\partial h} T
$$

A Figura 1 representa um ponto $P$ no espaço com coordenadas geodésicas $(h, \varphi, \lambda)$ referidos ao elipsoide e um ponto $P^{\prime}$ com coordenadas esféricas $(r, \varphi, \lambda)$ referidos à esfera, sendo $r=R$. Essa aproximação consiste em calcular o ponto $P^{\prime}$ como se fosse o ponto $P$. Isso pode ser conduzido a partir de uma linearização envolvendo o potencial perturbador e quantidades similares. Segundo Moritz 1980, o erro em função do não achatamento da esfera, que é de $0,3 \%$ pode ser negligenciado. Isso é usualmente permitido, por consequência, a aproximação esférica é utilizada na fórmula de Stokes (e.g. GOLDANI; QUINTAS, 2010) e (e.g. TOCHO et al., 2008), em soluções práticas do problema de Molodensky e também na colocação por mínimos quadrados (e.g. SOUZA; SÁ, 2008).

Figura 1 - Aproximação esférica.

Fonte: Adaptado de Moritz (1980).
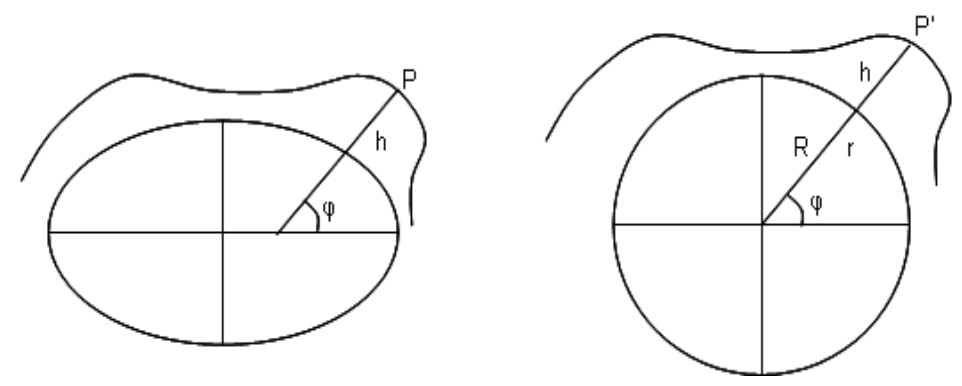

\subsection{A Formulação de Stokes}

A concepção clássica do PVCG é devida a Stokes. A fórmula ou integral de Stokes publicada em 1849 é considerada a de maior importância para a Geodésia. O problema clássico pode ser resolvido em termos do potencial perturbador por meio da referida integral, que numa aproximação esférica assim se expressa: (HOFMANN-WELLENHOF; MORITZ, 2005). 


$$
T=\frac{R}{4 \pi} \iint_{\sigma} \Delta g S(\psi) d \sigma
$$

onde o vetor anomalia de gravidade (Figura 2) é definido por:

$$
\Delta g=\vec{g}(P)-\vec{\gamma}(Q)
$$

$\vec{g}(P)=$ vetor gravidade num ponto $P$ da Terra real

$\vec{\gamma}(Q)=$ vetor gravidade num ponto $Q$ da Terra normal

Figura 2 - Vetores $\vec{g}$ e $\vec{\gamma}$.

Fonte: Blitzkow (1996).

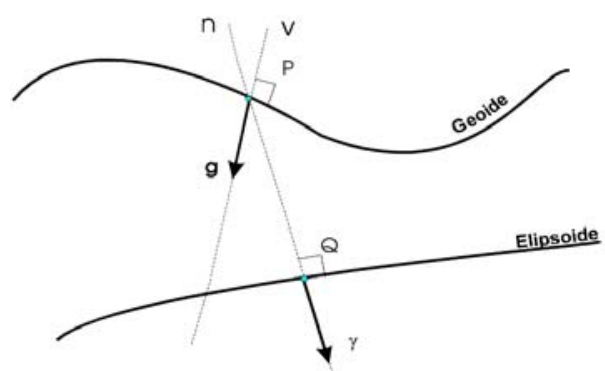

e a função de Stokes $S(\psi)$ é dada por:

$$
S(\psi)=\frac{1}{\operatorname{sen}\left(\frac{\psi}{2}\right)}-6 \operatorname{sen} \frac{\psi}{2}+1-5 \cos \psi-3 \cos \psi \ln \left(\operatorname{sen} \frac{\psi}{2}+\operatorname{sen}^{2} \frac{\psi}{2}\right)
$$

Considerando o teorema de Bruns:

$$
N=\frac{T}{\gamma}
$$

chega-se a:

$$
N=\frac{R}{4 \pi \gamma} \iint_{\sigma} \Delta g S(\psi) d \sigma
$$

Bol. Ciênc. Geod., sec. Comunicações/Trab. Técnicos, Curitiba, v. 17, nº 4, p.607-624, out-dez, 2011. 
onde $N$ é a ondulação geoidal.

A fórmula de Stokes na sua forma original equações (13) e (17) somente se aplica a um elipsoide de referência que (1) tenha o potencial de gravidade $U_{0}$ sobre o elipsoide igual ao potencial $W_{0}$ sobre o geoide; (2) cuja massa seja numericamente igual à massa da Terra; e (3) com mesma velocidade angular $(\omega)$ (HOFMANN-WELLENHOF; MORITZ, 2005).

\subsection{A Formulação de Molodensky}

O desconhecimento de um modelo de distribuição de densidade no interior da crosta terrestre levou Mikhail Molodensky, em 1945, a propor a superfície física como superfície limitante. Neste caso, substitui-se o conceito de geoide pelo quasegeoide. A anomalia de gravidade e as deflexões da vertical referem-se à superfície física e não mais à superfície geoidal. Além disso, as anomalias de altura tomam lugar antes ocupado pelas ondulações geoidais e a superfície limitante agora não possui um significado físico, não sendo equipotencial. A fórmula de Gauss, equação (18), bem como as identidades de Green estão entre as equações básicas na teoria do potencial e integram a fundamentação matemática para a formulação "moderna" do PVCG.

$$
\iiint_{v} d i v F d v=\iint_{S} F_{n} d S
$$

onde $v$ é o volume delimitado pela superfície $S, F_{n}$ é a projeção do vetor $F$ na superfície externa normal $n$ e $\operatorname{div} F$ é a chamada divergência do vetor $F$. 1967):

A partir das seguintes relações (maiores detalhes em HEISKANEN; MORITZ,

$$
F_{n}=\frac{\partial V}{\partial n} \text { e } F=\nabla V
$$

chega-se a integral de Gauss para o potencial:

$$
\iiint_{v} \nabla V d v=\iint_{S} \frac{\partial V}{\partial n} d S
$$

As identidades de Green são derivadas a partir da equação 18. A terceira identidade aplicada ao potencial de gravidade $W$, para um ponto sobre a superfície da Terra $S(p=2 \pi)$ e após algumas transformações (HEISKANEN; MORITZ, 1967) chega-se:

Bol. Ciênc. Geod., sec. Comunicações/Trab. Técnicos, Curitiba, v. 17, nº 4, p. 607-624, out-dez, 2011. 


$$
-2 \pi W+\iint_{S}\left[W \frac{\partial}{\partial n}\left(\frac{1}{l}\right)-\frac{1}{l} \frac{\partial W}{\partial n}\right] d S+2 \pi \omega^{2}\left(x^{2}+y^{2}\right)+2 \omega^{2} \iiint_{v} \frac{d v}{l^{\prime}}=0
$$

onde $S$ é a superfície física da Terra; $l$ é a distância entre o ponto fixo $P$ e o elemento da superfície $d S ; n$ a normal à superfície, orientada para fora de $S ;(\omega)$ é a velocidade angular; $l$ ' é a distância entre o ponto $P$ e o elemento do volume $d v$; $\partial W / \partial n$ a componente do vetor $\vec{g}$ normal a $S$.

Esse conceito retrata a determinação da forma da Terra $S$ a partir de medidas de $g$ e $W$. A combinação do nivelamento com medidas de gravidade em um ponto $P$ resulta no potencial de gravidade $W$, a menos de uma constante $W_{0}$.

$$
W=W_{0}-\int_{0}^{P} g d n
$$

As incógnitas a serem determinadas são $S$ e $W$, já que a componente normal $\partial W / \partial n$ do vetor gravidade $\vec{g}$ é determinada por medidas de gravimetria e sua direção estabelecida por coordenadas astronômicas. A equação (18) caracteriza-se por uma forma integral não-linear, impondo sua linearização para uma solução. Detalhes acerca da linearização podem ser encontrados em (HEISKANEN; MORITZ, 1967) e (BLITZKOW, 1996). Após o processo de linearização tem-se a seguinte equação (HEISKANEN; MORITZ, 1967):

$$
T-\frac{1}{2 \pi} \iint_{\Sigma}\left[\frac{\partial}{\partial n}\left(\frac{1}{l}\right)-\frac{1}{\gamma} \frac{\partial \gamma}{\partial h} \frac{\cos \beta}{l}\right] T d \Sigma=\frac{1}{2 \pi} \iint_{\Sigma}^{1} \frac{1}{l}\left[\Delta g-\gamma\left(\xi \tan \beta_{1}+\eta \tan \beta_{2}\right)\right] \cos \beta d \Sigma
$$

sendo $T$ a incógnita, $\xi$ e $\eta$ as componentes do desvio da vertical e $\beta_{1}$ e $\beta_{2}$ os ângulos de inclinação do terreno em relação à horizontal respectivamente segundo um perfil norte-sul e leste-oeste. $\mathrm{O}$ ângulo $\beta$ representa a inclinação máxima.

A equação (23) retrata o PVCG segundo a concepção de Molodensky. Para tanto, algumas considerações devem ser enfatizadas para a compreensão do problema. A Figura 3 ilustra as quantidades envolvidas no espaço real e de Helmert, onde SIE (Secondary Indirect Effects) são os efeitos indiretos secundários e PIE (Primary Indirect Effects) os efeitos indiretos primários. 
Figura 3 - Quantidades envolvidas no espaço real e de Helmert. Fonte: SHGEO software package (2005).

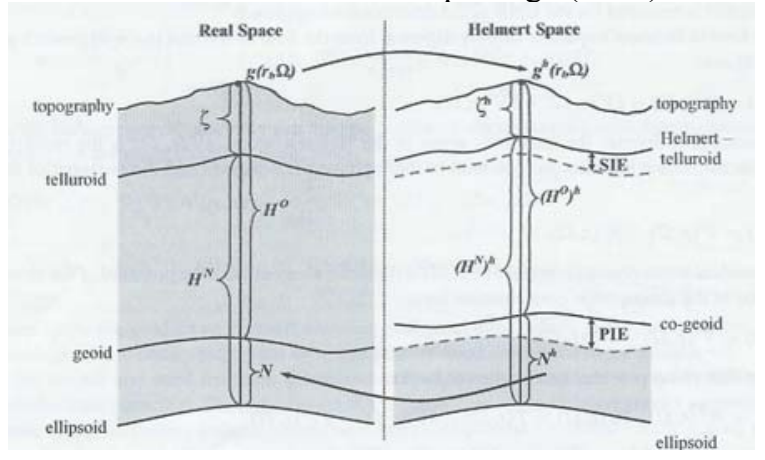

A anomalia de altura $\zeta$ pode ser considerada como a distância entre a superfície física e a superfície do teluroide. Ela é obtida por meio da seguinte equação:

$$
\zeta=\frac{T}{\gamma}
$$

A altitude normal $H_{\text {norm }}$ substitui a altitude ortométrica $H$ e a anomalia de altura $\zeta$ a ondulação geoidal $N$.

$$
h=H_{n o r m}+\zeta
$$

Além disso, a superfície de referência utilizada passa a ser o teluroide (HIRVONEN 1960, 1961). Trata-se de uma superfície cujo potencial normal $U$ em um dado ponto $Q$ é igual ao potencial de gravidade $W$ em todos os pontos $P$ da superfície física, $W_{P}=U_{Q}$. Sendo assim o referido teluroide passa a ser denominado de teluroide de Marussi. A altitude normal $H_{\text {norm }}$ é obtida a partir do número geopotencial $C$ :

$$
H_{\text {norm }}=\frac{C}{\gamma Q_{0}}\left[1+\left(1+f+m-2 f \operatorname{sen}^{2} \varphi\right) \frac{C}{a \gamma Q_{0}}+\left(\frac{C}{a \gamma Q_{0}}\right)^{2}\right]
$$

onde o potencial perturbador $T$ é obtido pela solução da equação (23) e a anomalia de gravidade pela (14). No caso da $\zeta$ ser plotada a partir do elipsoide, resultará em uma superfície denominada por Molodensky de quase-geoide. A partir da Figura 3 
obtêm-se as seguintes relações após considerar as curvaturas das diferentes linhas de campo:

$$
\begin{aligned}
& h=H_{n o r m}+\zeta \\
& h=H+N \\
& N-\zeta=H_{n o r m}-H
\end{aligned}
$$

A equação (23) pode ser aplicada ao geoide desde que um adequado processo de regularização cuide da remoção das massas externas do mesmo. Nestas condições, ao invés do teluroide adota-se como superfície aproximada a do elipsoide. Por outro lado, $\beta_{1}=\beta_{2}=\beta=0$ e $\partial / \partial n=\partial / \partial h$. Assim a equação (26) reduz-se a:

$$
T-\frac{1}{2 \pi} \iint_{\Sigma}\left[\frac{\partial}{\partial h}\left(\frac{1}{l}\right)-\frac{1}{\gamma} \frac{\partial \gamma}{\partial h} \frac{1}{l}\right] T d E=\frac{1}{2 \pi} \iint_{\Sigma} \frac{\Delta g}{l} d E
$$

O fato de não conter as componentes da deflexão $\xi$ e $\eta$, faz com que a equação (27) se torne mais simples que a (23). No caso de uma aproximação esférica, a solução da (27) é dada simplesmente pela integral de Stokes. Tal fato é verdadeiro, uma vez que a fórmula de Stokes expressa $T$ em termos de $\Delta g$.

\section{IMPORTANTES CONSIDERAÇÕES DO PVCG}

É sabido que a determinação do campo de gravidade da Terra a partir de dados terrestres está relacionada com a formulação dos problemas de valor de contorno. Várias versões do PVCG podem ser formuladas. Isso dependerá do tipo de dados, além do tipo e da quantidade de funções desconhecidas. Ademais, os dados ditos "clássicos", tais como, potencial de gravidade e coordenadas astronômicas combinados com dados de satélites, contribuem para novas versões do PVCG, como: misto, super determinado (HECK, 1997) e a solução de Brovar (HECK, 2011). Vale salientar que atualmente o PVCG ainda é fonte de estudos na Geodésia (VAL'KO, et al. 2008), (ARDALAN; KARIMI; BILKER-KOIVULA, 2010) e (FERREIRA, 2010). Nos próximos parágrafos será apresentada uma revisão conceitual dos três problemas clássicos: livre ou vetorial livre, escalar livre e fixo, além do PVCG gravimétrico fixo.

O modelo físico-matemático do PVCG pode ser formulado da seguinte forma (HECK, 1988), (BLITZKOW 1996), (HECK, 1997):

(1) A Terra é assumida como um corpo rígido, não deformável, com velocidade uniforme ao redor de um eixo fixo no espaço. Toda a massa de atração está localizada no interior da superfície de contorno, no caso, a superfície física. O potencial gravitacional newtoniano é gerado a partir da atração dos elementos de 
massa atuando sobre uma partícula teste, sendo este regular no infinito e que satisfaça a equação de Laplace no exterior às massas e a equação de Poisson no interior.

(2) A fim de descrever as posições no espaço, além de quantidades como o vetor gravidade, introduz-se à Terra um referencial fixo, com origem $\mathrm{O}$ no seu centro de massa. O eixo $x$ adjacente no plano do Meridiano Internacional de Referência, o eixo $z$ na direção do eixo de rotação orientado para o norte e por fim o eixo $y$ jacente no plano do equador completa o sistema dextrógiro. O vetor de gravidade $\vec{g}$ é representado pela equação:

$$
\frac{\partial W}{\partial x}=-\vec{g}
$$

(3) A superfície de contorno $S$ é suficientemente suave. Além disso, assume-se que tanto $W$ quanto $g$ sejam conhecidos sobre o contorno de forma contínua.

(4) Como incógnitas têm-se o potencial de gravidade $W$ no exterior das massas e a geometria da superfície de contorno $S$.

\subsection{O PVCG “Livre”}

Antes do advento das técnicas espaciais, era impossível determinar como um todo a geometria da superfície terrestre com alta precisão. O estabelecimento de redes globais tri-dimensionais a partir de medidas de distâncias, ângulos horizontais, azimutes e distâncias zenitais não era possível devido à dificuldade da refração vertical. Perante essa situação originou-se a formulação do PVCG Livre, também conhecido como problema de Molodensky.

Trata-se de um problema de valor de contorno não linear "livre" ou "vetorial livre", onde a "qualificação livre" está ligada ao fato do vetor posição ser uma incógnita. Não existe informação com relação à superfície de contorno. As observações que se têm são: $W, g, \Phi$ e $\Lambda$. Entretanto, a aplicação do PVCG livre possui a restrição de que o vetor posição está relacionado às observações que são basicamente coordenadas astronômicas; estas não possuem uma distribuição requerida e não atendem a precisão exigida (o erro é da ordem de $\sim 0,3$ ” $110 \mathrm{~m}$ ).

\subsection{O PVCG “Escalar livre”}

No passado a dificuldade na determinação das componentes horizontais do vetor posição levou ao desenvolvimento de um PVCG escalar livre. De fato, a informação proveniente da posição horizontal na superfície não pode ser extraída a partir de soluções do chamado PVCG clássico. Nos levantamentos geodésicos, as coordenadas geográficas $(\varphi, \lambda)$ eram derivadas dos métodos de poligonação e triangulação, a partir de medidas de ângulos e distâncias. A coordenada altimétrica (altitude elipsoidal), geralmente não era conhecida. Tal fato se deve à razão de não 
se realizar regularmente o nivelamento geométrico nas triangulações e poligonações. $\mathrm{O}$ que se executa é o nivelamento trigonométrico, obtendo assim a altitude ortométrica. Contudo, para se obter a altitude elipsoidal era necessário conhecer a altura geoidal, sendo que esta não figura no problema de Molodensky. Com isto a opção foi de se utilizar o PVCG escalar livre, uma vez que se conhecem as coordenadas geográficas em todos os pontos $P \in S$.

Ressaltando a diferença deste com o problema livre: neste, as coordenadas planimétricas da posição de $\mathrm{P}$ são conhecidas sobre a normal em $Q$, sendo somente necessário conhecer $(h(\varphi, \lambda))$ para fixar a superfície desconhecida. Além disso, para suprir a falta de informação, duas quantidades do campo de gravidade são medidas: $(W, g)$. Ao passo que no outro, não se conhece nada sobre a posição $P$, sendo que 4 grandezas são medidas: $W, g, \Phi$ e $\Lambda$.

As condições para a solução do PVCG escalar livre são assim enunciadas (HECK, 1989a):

“Combinando as forças, gravitacional e centrífuga, o potencial de gravidade $W$ é determinado no exterior de $S$.

$$
\nabla^{2} W=2 \omega^{2}
$$

E, além disso, deve-se admitir que $W$ (a menos de uma constante $W_{0}$ ) e o módulo do vetor gravidade $\|g\|$ sejam fornecidos na forma de uma função contínua, que seja suficientemente suave sobre a superfície $S$. Admite-se também que as coordenadas geográficas estejam vinculadas a um elipsoide de referência."

Seja um elipsoide, com mesma velocidade angular e mesma massa da terra real, na qual, o potencial $U_{0}$, constante sobre a superfície, seja igual ao potencial $W_{0}$ sobre a superfície geoidal e com seu centro no centro de massa da terra. Esta figura de referência é denominada de terra normal. A esta vincula-se o potencial de gravidade normal $U$ e o vetor gravidade normal $\gamma$.

Logo, esse PVC pode ser reformulado em termos de "pequenas" quantidades elegendo o potencial normal U e uma superfície limite conveniente $\Sigma$ com $Q \in \Sigma$. Os pontos $P \in S$ e $Q \in \Sigma$ devem satisfazer uma correspondência biunívoca. Uma definição do teluroide consistente com a informação que é inserida no PVCG escalar livre é dada fixando $P$ e $Q$ ao longo da mesma normal passando por $P$ (HECK, 1989a).

Consequentemente, o vetor da posição é expresso pela diferença de altitudes elipsoidais, chamada de "anomalia de altura".

$$
\zeta=h-H_{n o r m}
$$

Bol. Ciênc. Geod., sec. Comunicações/Trab. Técnicos, Curitiba, v. 17, no 4, p.607-624, out-dez, 2011. 
Analogamente, pode-se transformar a observação sobre a superfície física em "anomalia de potencial" $\Delta C$ e "anomalia de gravidade" $\Delta \gamma$. Para tanto, é necessário linearizar, pois ambas as anomalias dependem de funções não-lineares. A série de Taylor conduz à forma linearizada (HECK, 1989a). Outros detalhes acerca deste problema podem ser encontrados em (SACERDOTE; SANSÒ, 1986).

\subsection{O PVCG “Fixo"}

Essa alternativa do PVCG possui uma estreita relação com a era espacial. O advento da mesma propiciou a determinação de coordenadas em um referencial tridimensional geocêntrico. $\mathrm{O}$ surgimento de várias técnicas, aliado ao desenvolvimento tecnológico, colaboraram para que se alcançassem alta precisão no posicionamento. São algumas destas técnicas: LLR, SLR e GNSS. O completo conhecimento da geometria da superfície limite $(\varphi, \lambda, h)$ faz com que somente uma incógnita permaneça indeterminada, o potencial de gravidade externo às massas atrativas. Para tanto, assume-se que o módulo do vetor gravidade $\|g\|$ seja conhecido de forma contínua e suficientemente suave sobre toda a superfície $S$. O problema continua sendo não linear, sendo que a não linearidade se deve exclusivamente da norma a ser aplicada no vetor $g$. A escolha de um potencial de referência $U$ permite linearizar em função do potencial perturbador (HECK, 1991).

Um aspecto relevante tanto na escolha do PVCG escalar livre quanto no fixo é que, em ambos, a altitude empregada $H_{n o r m}$ não possui sentido físico e sim matemático. Isso implicará na determinação do quase-geoide e não do geoide. Em termos geométricos, a diferença entre geoide e quase-geoide é que o primeiro é uma superfície equipotencial do campo de gravidade, enquanto o segundo não. Tal escolha pode afetar trabalhos relacionados ao fluxo de água, como a transposição de rios, construção de hidrelétricas, etc.

\subsection{O PVCG “Gravimétrico Fixo”}

O lançamento de satélites com o objetivo de explorar o comportamento dos oceanos, sobretudo aqueles com tecnologia por radar, contribuiu de forma significativa para o estudo de mais um PVCG, o chamado problema gravimétrico fixo.

A altimetria por radar tem auxiliado nas pesquisas sobre circulação oceânica e suas variações espaços-temporais, sendo que vários satélites têm colaborado para isso. Os mais recentes, JASON-1, JASON-2 e ENVISAT (Environmental Satellite), possuem uma precisão da medida de 2,1 e $3 \mathrm{~cm}$, respectivamente (CHELTON et al., 2001). O princípio da medida está associado em determinar uma distância $R$ (Range) entre o satélite e a superfície do oceano. Para tanto, um pulso curto de radiação de microondas é transmitido do satélite até a superfície por um aparelho denominado de altímetro, sendo que parte dessa radiação é refletida de volta para o 
sensor. A distância é calculada a partir do tempo de percurso do pulso (31) (CHELTON et al., 2001).

$$
R=\hat{R}-\sum_{j} \Delta R_{j}
$$

onde $\hat{R}=c t / 2$ é a distância calculada baseada na velocidade da luz no vácuo $c$ e $t$ é o tempo de propagação (ida e volta); $\Delta R_{j},(\operatorname{com} \mathrm{j}=1, \ldots)$ são as correções para as várias componentes da refração atmosférica.

Desta forma, a altura da superfície do mar (Sea Surface Heigh) é determinada pela seguinte expressão:

$$
S S H=h-R
$$

em que $h$ é a altitude da órbita em relação a um elipsoide de referência específico do satélite altimétrico e $R$ é a distância entre o satélite e a superfície do mar. É evidente que várias correções precisam ser realizadas até obter o valor de uma medida final. Mais detalhes podem ser encontrados em (CHELTON et al., 2001). A SSH está relacionada ao geoide por meio da seguinte expressão:

$$
S S H=G+D T
$$

sendo $G$ o geoide e $D T$ a dinâmica da topografia.

Com as recentes missões espaciais de baixa órbita, CHAMP (CHAlleging Minisatellite Payload), GRACE (Gravity Recovery and Climate Experiment) e recentemente o GOCE (Gravity field and steady-state Ocean Circulation Explorer), a determinação do modelo geoidal nos oceanos tem sido cada vez mais precisa. A missão do satélite GOCE tem como objetivo determinar o geoide global com acurácia de 1-2 cm para uma resolução melhor que $100 \mathrm{~km}$.

A diferença deste para o PVCG fixo é a superfície de contorno. Além disso, a única incógnita é o potencial de gravidade externo às massas atrativas, que pode ser determinado por meio de medidas gravimétricas a partir do auxílio de navios ou aeronaves adaptados para esse tipo de levantamento. De fato, esse também é um problema não-linear. Após a linearização (ČUNDERLÍK et al., 2008), o resultado é um PVCG gravimétrico fixo de derivada oblíqua, sendo a direção da derivada na superfície de contorno idêntica com a direção normal do vetor de gravidade normal $\gamma=\operatorname{grad} U(\mathrm{HECK}, 1989 \mathrm{~b})$.

\section{SÍNTESE DO PVCG}

Apresentadas as duas formulações do PVCG e os aspectos relevantes do mesmo, a indagação que surge é saber qual a formulação a ser utilizada, ou melhor,

Bol. Ciênc. Geod., sec. Comunicações/Trab. Técnicos, Curitiba, v. 17, nº 4, p.607-624, out-dez, 2011. 
qual a superfície em que se trabalhará. A resposta está vinculada à possibilidade de dispor dos dados sobre a superfície conveniente. Será a partir dos mesmos que se poderá estabelecer subsídios para uma melhor determinação do geoide ou do quasegeoide. A Tabela 1 apresenta um comparativo entre os PVCG.

Tabela 1 - Grandezas do PVCG.

\begin{tabular}{l|c|c|l}
\hline PVCG & Conhecido & Desconhecido & Sup. de contorno \\
\hline Livre & $\Phi, \Lambda, W, g$ & $\varphi, \lambda, h$ & superfície física \\
\hline Escalar livre & $\varphi, \lambda, W, g$ & $h$ & superfície física \\
\hline Fixo & $\varphi, \lambda, h, W, g$ & - & superfície física \\
\hline Gravimétrico fixo & $\varphi, \lambda, H, W, g$ & - & superfície geoidal \\
\hline
\end{tabular}

Com relação às teorias de Stokes e Molodensky algumas considerações devem ser ressaltadas: Stokes propôs a determinação do geoide, enquanto Molodensky o quase-geoide; no primeiro é calculada a altura geoidal já, no segundo, a anomalia de altura. As anomalias de gravidade e as deflexões da vertical estão referidas ao nível dos mares (Stokes) e a superfície física (Molodensky). No primeiro, a superfície limitante envolve o campo de gravidade, enquanto no segundo não é uma superfície equipotencial. Por fim, a formulação de Stokes possui o problema do potencial perturbador não ser harmônico entre o geoide e a superfície da Terra, além da dificuldade das reduções topográficas.

Uma discussão do PVCG no contexto brasileiro e sul-americano diz respeito ao datum vertical em vista do Projeto SIRGAS (grupo de trabalho GTIII). Para a definição e a realização de um sistema de referência vertical global ou regional é necessário um modelo de gravidade da Terra baseado na combinação satelital e dados de gravimetria terrestre (marítima e aérea). O EGM2008 atende esse pressuposto. As inconsistências verticais entre os data locais provocados pelas tendências presentes nos dados de gravidade terrestre fazem com que o PVCG inclua valores de altitudes elipsoidais como uma nova observação, Além disso, são inseridos dados de aceleração da gravidade, deflexão da vertical e diferenças do geopotencial que permitem a unificação de um datum vertical global ou regional. Portanto, um sistema unificado deve compreender duas componentes: geométrica e física.

A componente com característica geométrica é dada por uma superfície de referência (elipsoide) e por altitudes elipsoidais $(h)$. É importante que as altitudes elipsoidais estejam de acordo com a realização de um sistema de referência (ITRS International Terrestrial Reference System e ITRF - International Terrestrial Reference Frame). Além disso, o elipsoide utilizado para derivar $h$ a partir das coordenadas geocêntricas $X, Y, Z$ tem que ser o mesmo empregado para estimar as ondulações geoidais ou anomalias de altura. Em relação à componente física, se a superfície de referência é baseada no geoide (altitudes ortométricas), deve-se 
reduzir as medidas gravimétricas para a superfície. Caso a superfície de referência se baseie no quase-geoide (altitudes normais), está será única e consistente, porém não será uma superfície equipotencial, não possuirá significado físico. Com o objetivo de apresentar uma definição consistente, livre de ambiguidades, e correta do ponto de vista teórico, a componente física do sistema de altitudes deve ser dada em termos do número geopotencial, i.e. a superfície de referência deve ter um valor fixo para $W_{0}$ e as coordenadas verticais deverão ter os números geopotenciais referidos ao mesmo $W_{0}$.

A determinação do número geopotencial absoluto é possível a partir de restrições adequadas. Para tanto, nessa aproximação considera-se o PVCG de duas maneiras: no oceano adota-se o PVCG gravimétrico fixo, na qual o número geopotencial estimado é aquele que se melhor adapta à superfície média do mar. Nos continentes emprega-se o PVCG escalar livre (aproximação de Molodensky), desde que os dados estejam referenciados a diferentes data verticais. Desta forma obtém-se tantos $W_{0, j}$ quanto sistemas de altitudes.

\section{REFERÊNCIAS BIBLIOGRÁFICAS}

ARDALAN, A. A.; KARIMI, R.; BILKER-KOIVULA, M. An overdetermined geodetic boundary value problem approach to telluroid and quasi-geoid computations. Journal of Geodesy, v. 84, n. 1, p. 97-104, 2010.

BLITZKOW, D. O problema do valor de contorno da geodésia: resultados práticos para a América do Sul. Tese (Livre Docência) - Escola Politécnica da Universidade de São Paulo, São Paulo, 1996.

BLITZKOW, D.; CAMPOS, I. de O.; FREITAS, S.R.C. Altitude: o que interessa e como equacionar? Informações Espaciais II - Notas de aula, Laboratório de Topografia e Geodésia - Escola Politécnica de São Paulo, São Paulo, 2007.

CHELTON, D. B.; RIES, J. C.; HAINES, B. J.; FU, L.L.; CALLAHAN, P. S. Satellite altimetry and earth sciences, $A$ handbook of techniques and applications: Satellite altimetry. In: FU, L.L.; CAZENAVE, A. (Ed.). San Diego: Academic Press, p. 1-122, 2001.

ČUNDERLÍK, R.; MIKULA, K.; MOJZEŠ, M. Numerical solution of the linearized fixed gravimetric boundary-value problem. Journal of Geodesy, v. 82, n. 1, p. 15-29, 2008.

FERREIRA, V. G.; FREITAS, S R. C. Análise do termo de primeira ordem das séries de Molodenskii para o problema de valor de contorno da geodésia. Boletim de Ciências Geodésicas, v. 16, n. 4, p. 557-574, out-dez, 2010.

FREITAS, S. R. C.; BLITZKOW, D. Altitudes e Geopotencial. IGeS Bulletin N.9International Geoid Service, Junho 1999, Milão, p. 47-62, 1999.

GEMAEL, C. Introdução a Geodésia Física. Curitiba: Editora UFPR, 1999.

GOLDANI, D.; QUINTAS, M. C. L. Um modelo geoidal para o estado do Paraná: aplicações da integral de Stokes e de um valor do geopotencial. Boletim de Ciências Geodésicas, v. 16, n. 1, p. 105-124, jan-mar, 2010. 
HECK, B. The non-linear geodetic boundary value problem in quadratic approximation. Manuscripta Geodaetica, v. 13, p. 337-348, 1988.

. A contribution to the scalar free boundary value problem of physical geodesy. Manuscripta Geodaetica, v.14, p. 87-99, 1989a.

. On the non-linear geodetic boundary value problem for a fixed boundary surface. Bulletin Géodésique v.63, p. 57-67, 1989b.

. On the linearized boundary value problems of physical geodesy. Ohio State University, Report 407. Columbus, 1991.

. Formulation and linearization of boundary value problems: from observables to a mathematical model. In: SANSÒ, F.; RUMMEL, R. (Eds.). Geodetic boundary value problems in view of the one centimeter geoid. Berlin: Springer, (Lectures Notes in Earth Sciences, v.65) p. 121-160, 1997.

. A Brovar-type solution of the fixed geodetic boundary-value problem. Studia Geophysica et Geodaetica v. 55, n. 3 p. 441-454, 2011.

HEISKANEN, W. A.; MORITZ, H. Physical geodesy. San Francisco: Freeman and Co., 1967.

HIRVONEN, R.A. New theory of gravimetric geodesy. Publications of the Isostatic Institute of the International Association of Geodesy, Helsinki, v. 32, 1960.

. The reformation of geodesy. Journal of Geophysical Research, v. 66, p. 1471-1478, 1961.

HOFMANN-WELLENHOF, B.; MORITZ, H. Physical geodesy. $2^{\text {nd }}$ Edition, New York: Springer. 2005.

MORITZ, H. Advanced Physical Geodesy. Karlsruhe: Wichman Verlag. 1980.

SACERDOTE, F.; SANSÒ, F. The scalar boundary value problem of physical geodesy. Manuscripta Geodaetica, v. 11, p. 15-28, 1986.

SHGEO SOFTWARE PACKAGE. Reference Manual 1. Fredericton, New Brunswick, Canada, 2005. 36 p.

SOUZA, S. F.; SÁ, N. C. Avaliação de modelos geoidais gravimétricos determinados com funções covariâncias planas e esféricas (estudo de caso: estado de São Paulo e adjacências). Boletim de Ciências Geodésicas, v. 14, n. 3, p. 331-349, jul-set, 2008.

TOCHO, C.; MIRANDA, S.; PACINO, M. C.; FORSBERG, R. Preliminary geoid model in San Juan province: a case study in the Andes. Boletim de Ciências Geodésicas, v. 14, n. 3, p. 316-330, jul-set, 2008.

VAL'KO, M.; MOJZES, M.; JANÁK, J.; PAPCO, J. Comparison of two different solutions to Molodensky's G1 term. Studia Geophysica et Geodaetica, v. 52, n. 1, p. 71-86, 2008.

(Recebido em agosto de 2011. Aceito em outubro de 2011.) 